\title{
Characterizing White Matter Tract Organization in Polymicrogyria and Lissencephaly: A Multifiber Diffusion MRI Modeling and Tractography Study
}

\author{
(D) F. Arrigoni, (DD. Peruzzo, (D). Mandelstam, (D). Amorosino, (DD. Redaelli, (D) R. Romaniello, (D) R. Leventer, (D) Rorgatti,
}

(D) M. Seal, and (D).Y.-M. Yang

ir

\begin{abstract}
BACKGROUND AND PURPOSE: Polymicrogyria and lissencephaly may be associated with abnormal organization of the undelying white matter tracts that have been rarely investigated so far. Our aim was to characterize white matter tract organization in polymicrogyria and lissencephaly using constrained spherical deconvolution, a multifiber diffusion MR imaging modeling technique for white matter tractography reconstruction.
\end{abstract}

MATERIALS AND METHODS: We retrospectively reviewed 50 patients (mean age, $8.3 \pm 5.4$ years; range, 1.4-21.2 years; 27 males) with different polymicrogyria $(n=42)$ and lissencephaly $(n=8)$ subtypes. The fiber direction-encoded color maps and 6 different white matter tracts reconstructed from each patient were visually compared with corresponding images reconstructed from 7 agematched, healthy control WM templates. Each white matter tract was assessed by 2 experienced pediatric neuroradiologists and scored in consensus on the basis of the severity of the structural abnormality, ranging from the white matter tracts being absent to thickened. The results were summarized by different polymicrogyria and lissencephaly subgroups.

RESULTS: More abnormal-appearing white matter tracts were identified in patients with lissencephaly compared with those with polymicrogyria (79.2\% versus $37.3 \%$ ). In lissencephaly, structural abnormalities were identified in all studied white matter tracts. In polymicrogyria, the more frequently affected white matter tracts were the cingulum, superior longitudinal fasciculus, inferior longitudinal fasciculus, and optic radiation-posterior corona radiata. The severity of superior longitudinal fasciculus and cingulum abnormalities was associated with the polymicrogyria distribution and extent. A thickened superior fronto-occipital fasciculus was demonstrated in 3 patients.

CONCLUSIONS: We demonstrated a range of white matter tract structural abnormalities in patients with polymicrogyria and lissencephaly. The patterns of white matter tract involvement are related to polymicrogyria and lissencephaly subgroups, distribution, and, possibly, their underlying etiologies.

ABBREVIATIONS: $C G=$ cingulum; $C M V=$ cytomegalovirus; $C S D=$ constrained spherical deconvolution; $D E C=$ direction-encoded color; $d M R I=$ diffusion $\mathrm{MRI} ; \mathrm{FOD}=$ fiber orientation distribution; $\mathrm{HARDI}=$ high angular resolution diffusion imaging; IFOF $=$ inferior fronto-occipital fasciculus; ILF = inferior longitudinal fasciculus; LIS = lissencephaly; MCD = malformation of cortical development; OR-PCR = optic radiation-posterior corona radiata; PMG = polymicrogyria; $\mathrm{SBH}=$ subcortical band heterotopia; SFOF $=$ superior fronto-occipital fasciculus/Muratoff bundle; SLF $=$ superior longitudinal fasciculus; WMT $=$ white matter tract

Received March 6, 2020; accepted after revision May 11.

From the Scientific Institute, IRCCS E. Medea (F.A., D.P., G.A., D.R., R.R), Bosisio Parini, Italy; Murdoch Children's Research Institute (S.M., R.L., M.S., J.Y.-M.Y.), Parkville, Australia; Royal Children's Hospital (S.M., R.L.), Parkville, Australia; Neuroscience Advanced Clinical Imaging Suite (NACIS) (J.Y.-M.Y.), Department of Neurosurgery, The Royal Children's Hospital, Victoria, Australia; University of Melbourne (S.M., R.L., M.S., J.Y.-M.Y.), Parkville, Australia; Florey Institute of Neuroscience and Mental Health (S.M.), Parkville, Australia; Bruno Kessler Foundation (G.A.), Trento, Italy; University of Trento, Center for Mind/Brain Sciences (G.A.), Rovereto, Italy; Istituto di ricovero e cura a carattere scientifico Mondino Foundation (R.B.), Pavia, Italy; and University of Pavia (R.B.), Pavia, Italy.

This study was funded by the Italian Ministry of Health (Ricerca Corrente 2018 and 2019 to Drs Arrigoni and Borgatti). Dr Arrigoni was also funded by the Australian Government through the Endeavor Research Fellowship program 2018 and by "Fondazione Banca del Monte di Lombardia." Dr Yang was funded by the Royal Columbian Hospital Foundation (RCH-1000). The research was supported by the Royal Children's Hospital Foundation, Murdoch Children's Research Institute, The University of Melbourne Department of Pediatrics, and the Victorian Government's Operational Infrastructure Support Program. Data used in the preparation of this article were obtained from the Pediatric MRI Data Repository created by the National Institutes of Health MRI Study of Normal Brain Development. This is a multisite, longitudinal study of typically developing children, from ages neonate through young adulthood, conducted by the Brain Development Cooperative Group and supported by the National Institute of Child Health and Human Development, the National Institute on Drug Abuse, the National Institute of Mental Health, and the National Institute of Neurologic Disorders and Stroke (contract Nos. NO1HD02-3343, N01-MH9-0002, and N01-NS-9-2314, -2315, -2316, -2317, -2319 and -2320)

This article reflects the views of the authors and may not reflect the opinions or views of the National Institutes of Health.

Data previously presented, in part, as posters at: Annual Meeting of the Organization for Human Brain Mapping, June 9-13, 2019, Rome, Italy; and the Annual Meeting of the European Society of Neuroradiology, September 19-22, 2019, Oslo, Norway.

Please address correspondence to Filippo Arrigoni, MD, Neuroimaging Lab, Scientific Institute IRCCS E. Medea, Via don Luigi Monza 20, 23842, Bosisio Parini, Italy; e-mail: filippo.arrigoni@lanostrafamiglia.it

\footnotetext{
- Indicates open access to non-subscribers at www.ajnr.org

Indicates article with supplemental on-line appendix and tables.

Indicates article with supplemental on-line photos.

http://dx.doi.org/10.3174/ajnr.A6646
} 
M alformations of cortical development (MCDs) are a spectrum of brain disorders characterized by neuronal proliferation, neuronal migration, or postmigrational cortical organization abnormalities occurring during the prenatal period. ${ }^{1}$ Polymicrogyria (PMG) is a class of MCD, characterized by cortical overfolding and increased gyral and sulcal numbers. Pachygyria and lissencephaly (LIS) are characterized by cortical underfolding and reduced gyral and sulcal numbers. ${ }^{1}$

Compared with cortical abnormalities, the white matter tract (WMT) organization in MCD is less well-investigated. Conventional structural MR imaging, such as T1WI and T2WI, is unable to demonstrate the WMT anatomy. Diffusion MR imaging (dMRI) tractography is currently the only noninvasive imaging technique that can estimate, in vivo, WMTs of the human brain. $^{2}$ Existing dMRI tractography studies in non-MCD brain malformations have demonstrated different patterns of aberrant WM connections in patients sharing similar-appearing malformations. These studies help advance our understanding about the disease pathologic mechanisms ${ }^{3-7}$ and may aid radiology practice. . $^{8}$

Previous dMRI tractography studies in LIS and PMG included only small case series or single case reports. ${ }^{10-17}$ These studies typically investigated the superior longitudinal fasciculus (SLF), corpus callosum, and corticospinal tract and interrogated the association between WMT abnormalities and language or motor function. No studies additionally characterized structural abnormalities in other WMTs, such as the inferior fronto-occipital fasciculus (IFOF), inferior longitudinal fasciculus (ILF), and optic radiation-posterior corona radiata (OR-PCR). These WMTs are components of the language and visual-spatial networks. ${ }^{18}$ Additionally, diffusion tensor imaging was used to model axonal fiber orientations in these studies-a technique that is limited in resolving fiber orientations in regions containing crossing fibers or other complex multifiber arrangements. Advanced dMRI acquisition schemes, such as high angular resolution diffusion imaging (HARDI), ${ }^{19}$ and multifiber modeling techniques, such as constrained spherical deconvolution (CSD), ${ }^{20}$ improve the accuracy of WM modeling in both healthy and pathologic brains, ${ }^{21}$ but they have not been applied to study WMT organization in LIS and PMG.

The aim of this study was to investigate the appearance of 6 major WMTs in LIS and PMG using tractography reconstructed from the CSD modeling technique.

\section{MATERIALS AND METHODS Study Population}

The MR imaging data were retrospectively selected from 2 tertiary pediatric referral hospitals: the E. Medea Institute (Bosisio Parini, Italy) and the Royal Children's Hospital (Melbourne, Australia). Both hospitals' ethics committee approved the study. The Royal Children's Hospital Ethics Committee approved the study as a clinical audit and determined that informed consent was unnecessary. Written inform consent was obtained from all patients and/or their legal guardians from the E. Medea Institute.
Inclusion criteria were the following: 1) radiologic diagnosis of PMG or LIS spectrum (agyria, pachygyria and subcortical band heterotopia $[\mathrm{SBH}])^{1}$ made on the basis of screening the hospital records and confirmed by an experienced pediatric neuoradiologist (F.A.), who reviewed all MR imaging data; and 2) a $3 \mathrm{~T}$ MR imaging study including a 3D structural T1weighted sequence and a DWI acquisition with $\geq 32$ diffusionweighted directions and a b-value $\geq 1000 \mathrm{~s} / \mathrm{mm}^{2}$.

MR imaging data of typically developing children obtained from the Pediatric MRI Data Repository were used as study control (NIH MRI Study of Normal Brain Development; https://neuroscienceblueprint.nih.gov/resourcestools/nih-mri-study-normal-brain-development). ${ }^{22}$ The MR imaging sequence details are summarized in On-line Table 1.

\section{DWI Processing and Tractography Reconstruction}

The DWI data were first preprocessed with Tortoise software (Version 3.1.4; https://tortoise.nibib.nih.gov/), ${ }^{23}$ corrected for thermal noise, Gibbs ringing artifacts, motion and eddy current distortions, and $\mathrm{B}_{1}$ bias field inhomogeneities. The EPI geometric distortion was corrected using a reversed phase-polarity DWI series or $b=0 \mathrm{~s} / \mathrm{mm}^{2}$ images.

Voxelwise WM fiber orientation distribution (FOD) was estimated on the basis of the multitissue CSD model using the MRtrix 3.0 software (www.mrtrix3.org) ( $\operatorname{lmax}=4$ for 32-direction data; $\operatorname{lmax}=8$ for 60 -direction data). ${ }^{24}$ FOD-based direction-encoded color (DEC) maps were generated using the conventional orientation labels.

Due to a propensity for a noisier FOD modeling in most cases with $b=1100 \mathrm{~s} / \mathrm{mm}^{2}$ data, we elected to perform tractography reconstructions using a deterministic tracking algorithm and a conservative FOD amplitude cutoff (0.1) to minimize false-positive streamline reconstructions; 5000 streamlines were retained per WMT, and other default-tracking parameters were used. ROIs used for in vivo WMT dissection were placed on the basis of a priori anatomic knowledge. ${ }^{25} \mathrm{We}$ reconstructed the following WMTs: cingulum (CG), IFOF, ILF, ORPCR, superior fronto-occipital fasciculus/Muratoff bundle (SFOF), and SLF.

To investigate the interscanner variance in the reconstructed tractography images due to differences in DWI acquisition schemes among the patients and controls, we conducted a preliminary analysis by scanning a single healthy subject using the 3 different study DWI acquisition schemes (see the On-line Appendix and On-line Figs 1 and 2 for more details). The reconstructed DEC maps and tractography images were visually comparable between different datasets, confirming that our study approach was suitable for semiquantitative assessment of the WMT morphology (see below).

\section{Age-Matched Typically Developing Brain Template}

Seven age-specific T1WI and WM FOD templates were generated from healthy controls. On-line Table 2 summarizes the age groups and template details. The FOD templates were created using the group-averaged multitissue response function from each age group. ${ }^{26}$ Each FOD template was optimized through 12 
Table 1: Study patients by the MCD subgroups and their conventional structural MR imaging findings

\begin{tabular}{|c|c|c|c|c|c|c|c|c|c|}
\hline \multirow[b]{2}{*}{ MCD Subgroups/Variants } & \multirow{2}{*}{$\begin{array}{l}\text { No. of } \\
\text { Cases }\end{array}$} & \multirow{2}{*}{$\begin{array}{l}\text { Unilateral } \\
\text { MCD }\end{array}$} & \multirow{2}{*}{$\begin{array}{c}\text { Prenatal } \\
\text { CMV } \\
\text { Infection }\end{array}$} & \multicolumn{6}{|c|}{ Associated Brain Anomalies } \\
\hline & & & & Ventricles & BGT & Hippocampus & Brain Stem & Cerebellum & $\mathrm{CC}$ \\
\hline \multicolumn{10}{|l|}{ PMG } \\
\hline Peri-Sylvian & 16 & 4 & 1 & 9 & 3 & 5 & 2 & 4 & 5 \\
\hline Frontoparietal & 7 & 2 & 4 & 2 & 0 & 0 & 2 & 2 & 2 \\
\hline Generalized & 4 & 0 & 0 & 4 & 1 & 4 & 0 & 2 & 3 \\
\hline Focal $^{a}$ & 7 & 2 & 0 & 4 & 2 & 3 & 2 & 3 & 5 \\
\hline Parieto-occipital & 1 & 1 & 0 & 1 & 0 & 0 & 0 & 0 & 1 \\
\hline Multifocal & 6 & 2 & 0 & 3 & 0 & 3 & 0 & 1 & 5 \\
\hline With schizencephaly & 1 & 0 & 0 & 1 & 1 & 1 & 1 & 1 & 1 \\
\hline Total & 42 & 11 & 5 & 24 & 7 & 16 & 7 & 13 & 22 \\
\hline \multicolumn{10}{|l|}{ LIS $^{\mathrm{b}}$} \\
\hline Pachygyria with SBH & 5 & 0 & 0 & 5 & 2 & 3 & 2 & 2 & 4 \\
\hline Pachygyria without SBH & 1 & 0 & 0 & 0 & 1 & 2 & 1 & 2 & 2 \\
\hline SBH without pachygyria & 2 & 0 & 0 & 1 & 0 & 0 & 0 & 0 & 2 \\
\hline Total & 8 & 0 & 0 & 6 & 3 & 5 & 3 & 4 & 8 \\
\hline
\end{tabular}

Note:-BGT indicates basal ganglia and thalami; CC, corpu callosum.

${ }^{a}$ All located in frontal lobe.

${ }^{\mathrm{b}}$ All LIS cases were bilateral.

Table 2: Summary of the white matter tract appearance in all study patients presented by each MCD subgroup ${ }^{a}$

\begin{tabular}{|c|c|c|c|c|c|c|c|}
\hline MCD Subgroups & No. of Patients & SLF & CG & SFOF & IFOF & OR-PCR & ILF \\
\hline Peri-Sylvian PMG & 16 & $8 / 16 / 0 / 8$ & $27 / 5 / 0 / 0$ & $31 / 1 / 0 / 0$ & $27 / 5 / 0 / 0$ & $20 / 12 / 0 / 0$ & $21 / 11 / 0 / 0$ \\
\hline Frontoparietal PMG & 7 & $4 / 6 / 0 / 4$ & $13 / 1 / 0 / 0$ & $12 / 2 / 0 / 0$ & $12 / 2 / 0 / 0$ & $6 / 8 / 0 / 0$ & $7 / 7 / 0 / 0$ \\
\hline Frontal PMG & 7 & $3 / 10 / 0 / 1$ & $5 / 9 / 0 / 0$ & $12 / 1 / 0 / 1$ & $12 / 2 / 0 / 0$ & $10 / 4 / 0 / 0$ & $11 / 3 / 0 / 0$ \\
\hline Generalized PMG & 4 & $0 / 2 / 0 / 6$ & $1 / 5 / 0 / 2$ & $2 / 2 / 0 / 4$ & $2 / 6 / 0 / 0$ & $0 / 8 / 0 / 0$ & $0 / 8 / 0 / 0$ \\
\hline Multifocal PMG & 6 & $9 / 3 / 0 / 0$ & $7 / 5 / 0 / 0$ & $10 / 0 / 0 / 2$ & $12 / 0 / 0 / 0$ & $9 / 3 / 0 / 0$ & $11 / 1 / 0 / 0$ \\
\hline Parieto-occipital PMG & 1 & $2 / 0 / 0 / 0$ & $2 / 0 / 0 / 0$ & $2 / 0 / 0 / 0$ & $2 / 0 / 0 / 0$ & $1 / 1 / 0 / 0$ & $2 / 0 / 0 / 0$ \\
\hline PMG and schizenchephaly & 1 & $0 / 0 / 0 / 2$ & $0 / 2 / 0 / 0$ & $0 / 0 / 1 / 1$ & $0 / 2 / 0 / 0$ & $0 / 2 / 0 / 0$ & $0 / 2 / 0 / 0$ \\
\hline Pachygyria with SBH & 5 & $0 / 6 / 0 / 4$ & $2 / 8 / 0 / 0$ & $2 / 4 / 4 / 0$ & $2 / 8 / 0 / 0$ & $0 / 10 / 0 / 0$ & $0 / 10 / 0 / 0$ \\
\hline SBH (no pachygyria) & 2 & $0 / 4 / 0 / 0$ & $2 / 2 / 0 / 0$ & $4 / 0 / 0 / 0$ & $2 / 2 / 0 / 0$ & $0 / 4 / 0 / 0$ & $0 / 4 / 0 / 0$ \\
\hline Pachygyria (no SBH) & 1 & $0 / 2 / 0 / 0$ & $0 / 2 / 0 / 0$ & $2 / 0 / 0 / 0$ & $0 / 2 / 0 / 0$ & $2 / 0 / 0 / 0$ & $2 / 0 / 0 / 0$ \\
\hline All PMG & 42 & $27 / 36 / 0 / 21$ & $55 / 27 / 0 / 2$ & $69 / 6 / 1 / 8$ & $67 / 17 / 0 / 0$ & $46 / 38 / 0 / 0$ & $52 / 32 / 0 / 0$ \\
\hline All LIS & 8 & $0 / 12 / 0 / 4$ & $4 / 12 / 0 / 0$ & $8 / 4 / 4 / 0$ & $4 / 12 / 0 / 0$ & $2 / 14 / 0 / 0$ & $2 / 14 / 0 / 0$ \\
\hline
\end{tabular}

${ }^{\mathrm{a}}$ For each white matter tract, the number of tract appearances classified as grades I/IIA/IIB /III is reported.

affine and 16 nonlinear iterations of FOD registration. Each T1WI template was then linearly coregistered to the FOD template. $^{27}$

\section{Data Analysis: A Semiquantitative WM Tract Analysis}

Both the tractography reconstruction and the tract appearance on DEC maps for each patient were compared with the corresponding age-matched typically developing WM-FOD template. The WMTs were assessed independently by 2 experienced pediatric neuoradiologists (F.A. and S.M.) and scored in consensus using a modified version of a semiquantitative scoring system previously used to grade WMT abnormalities in other MCDs: $:^{13,28}$

- Grade I (normal): WMTs with similar size and geometry compared with the healthy controls

- Grade IIA (irregular): WMTs characterized by at least 1 of the following features: reduced size, displaced fibers, or distorted geometry

- Grade IIB (thick): WMTs with increased size compared with those in the healthy controls

- Grade III (absent): failed tractography reconstruction and no recognizable WMTs on the DEC maps
The results were summarized by the different PMG and LIS subgroups. ${ }^{1}$

\section{RESULTS}

Fifty patients were included in this study (mean age, $8.3 \pm$ 5.4 years; range, 1.4-21.2 years; 27 males). Forty-two patients had PMG, and 8 patients had the LIS spectrum. PMG was unilateral in 11 patients. The main associated brain structural abnormalities included the following: mild-to-severe ventricular dilation (30 cases), hippocampal malrotation or asymmetry (21 cases), basal ganglia and thalami dysmorphism (10 cases), brain stem hypoplasia, asymmetry, and malformations (10 cases), cerebellar hypoplasia/atrophy and dysplasia (17 cases), and corpus callosum anomalies (30 cases).

A genetic diagnosis was confirmed in 11 cases (see On-line Table 3 for details). Five patients had a proved congenital cytomegalovirus (CMV) infection (10 of 50 patients suspicious for congenital CMV were tested). Table 1 summarizes the patient demographics and MCD characteristics. ${ }^{1}$ Table 2 summarizes the WMT analysis results.

The patients with LIS had more WMT abnormalities than those with PMG (percentage of abnormal tracts in LIS and PMG: 


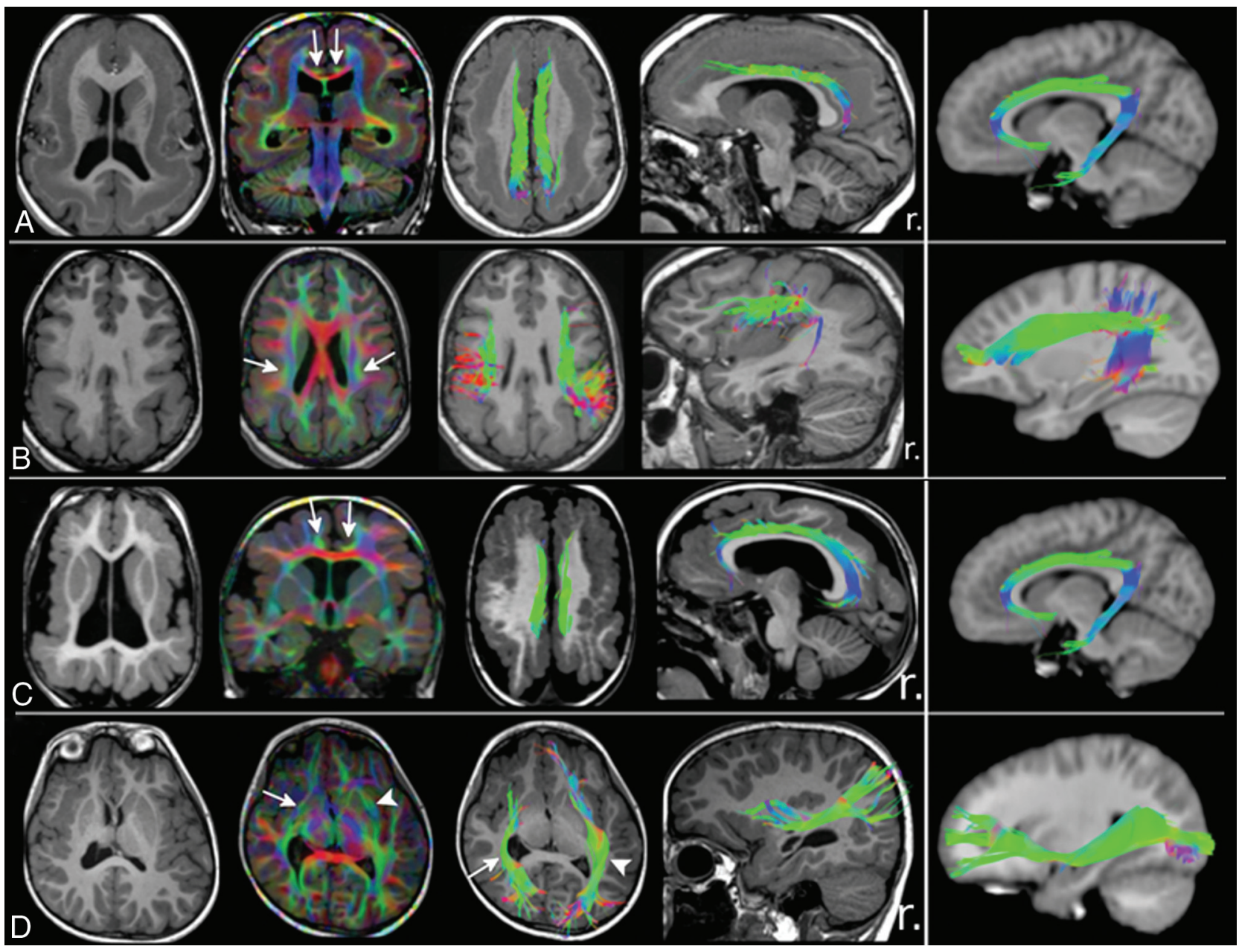

FIG 1. Tractography findings in selected patients with lissencephaly and polymicrogyria. TIWI, direction-encoded color maps, and tractography reconstructions in 2 patients with LIS $(A$ and $B)$ and 2 with PMG $(C$ and $D)$ are shown. For each patient, a comparable tractography reconstruction from the age-matched healthy control template is shown in the last column for comparison. $A$, An irregular (shorter and smaller) bilateral cingulum (arrows on DEC map) in a patient with pachygyria and thick subcortical band heterotopia. B, A bilateral, irregular (smaller and distorted) superior longitudinal fasciculus (arrows on DEC map) in a patient with posterior-quadrant pachygyria and a thick SBH. C, Bilateral irregular (smaller and thinner) CG (arrows on DEC map) in a patient with generalized PMG. D, An irregular (shorter and distorted) right inferior frontooccipital fasciculus (IFOF; arrows) in a patient with unilateral right peri-Sylvian PMG. The left IFOF (arrowheads) has a normal appearance. r. indicates right.

79.2\% versus $37.3 \%$ ). In LIS, different grades of tract abnormalities were identified in every WMT. In PMG, the more frequently affected WMTs were the CG, ILF, PCR-OR, and SLF (Fig 1 and On-line Fig 3).

The extent of WMT abnormalities in PMG was associated with the severity and distribution/location of the cortical abnormalities. For example, in generalized PMG, most studied WMTs had an abnormal appearance. The SLF was absent in the majority of these patients (75\%). The SLF was most frequently affected in patients with peri-Sylvian PMG. In focal (frontal) PMG, most of the WMT abnormalities involved the CG and SLF (both have frontal fiber projections), not the OR-PCR and ILF (neither has frontal fiber projections). In the peri-Sylvian PMG subgroup, normal-appearing SLFs were observed in focal opercular and/or insular PMG, irregular-appearing SLFs were observed in more extensive peri-Sylvian PMG, and the SLFs were absent in diffuse peri-Sylvian PMG cases with malformations extending into the adjacent frontal/parietal lobes (Fig 2 and On-line Fig 4).
Irregular-appearing SLFs were identified in 4 frontoparietal and 1 diffuse peri-Sylvian PMG with proved congenital CMV infection (On-line Fig 5). Thickened SFOFs were identified in 2 patients with LIS with pachygyria and SBH and 1 patient with PMG with schizencephaly (Fig 3).

\section{DISCUSSION}

In this qualitative dMRI tractography study, we demonstrated abnormal patterns of WMT organization in a large cohort of patients with complex MCDs. The incorporation of CSD, an advanced multifiber WM model, is unique and a strength to this study, compared with the other MCD tractography studies, using the single-fiber, diffusion tensor imaging model. The use of this higher-order diffusion model helps improve the anatomic accuracy of the tractography reconstructions by reducing both false (premature) terminations and inaccurate streamline propagation over crossing-fiber WM regions. ${ }^{20,21}$ Our results 

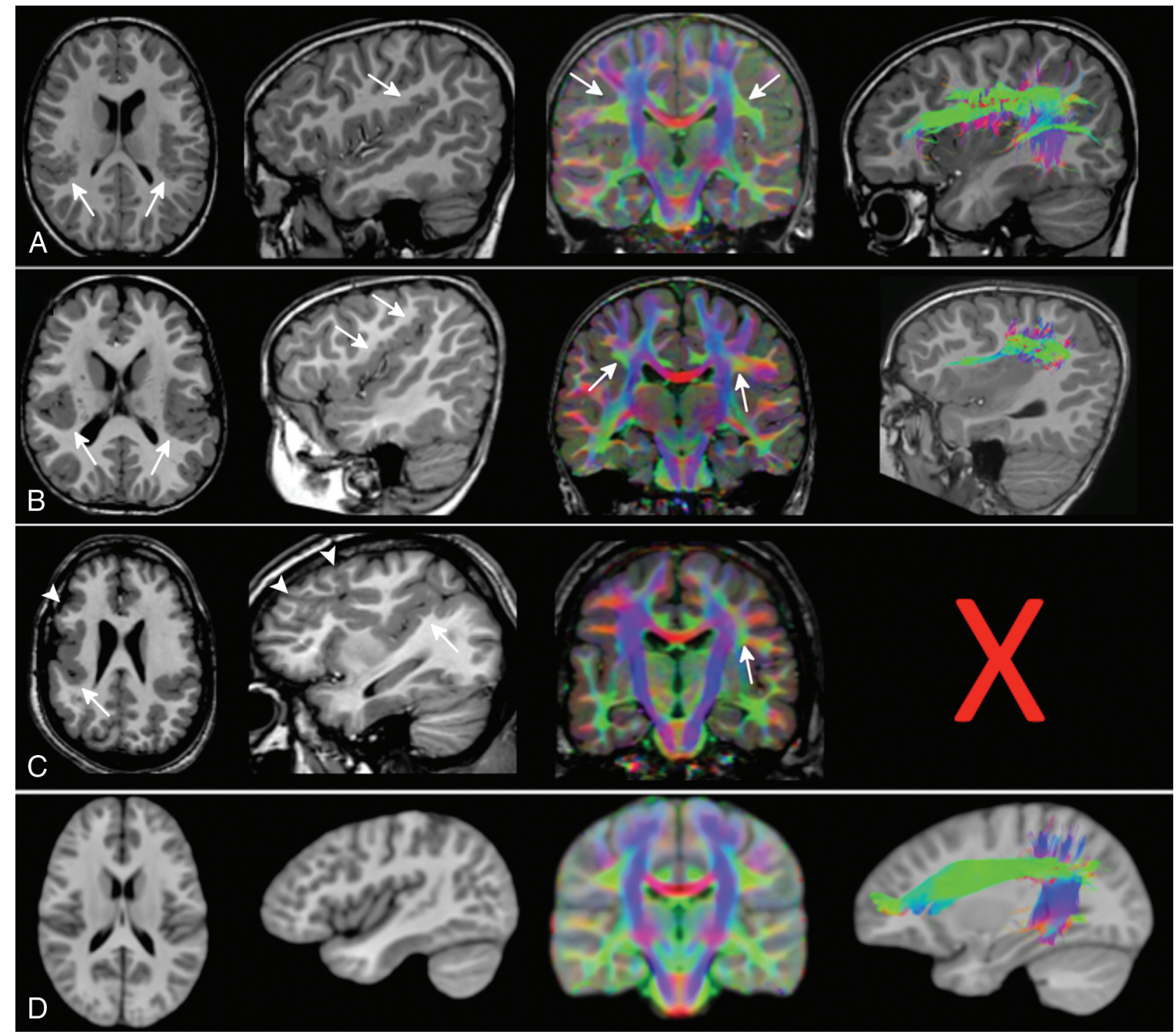

FIG 2. The superior longitudinal fasciculus involvement in peri-Sylvian polymicrogyria. TIWI, direction-encoded color maps, and tractography reconstructions of the SLF in 3 patients with exemplary peri-Sylvian PMG $(A-C)$. Sagittal TT-weighted images and tractography refer to the right hemisphere. A comparable SLF tractography reconstruction from the age-matched healthy control template is shown in the last row for comparison (D). Patient A has bilateral focal peri-Sylvian PMG in the opercular regions (arrows on TIWI). Both SLFs look normal on both the DEC map (arrows on DEC map) and tractography reconstruction. Patient B has bilateral diffuse peri-Sylvian PMG (arrows on TTWI). Both SLFs (arrows on DEC maps) are irregular in appearance. The right SLF tractography is notably shorter and smaller compared with the right SLF from a control. Patient $C$ has bilateral diffuse peri-Sylvian PMG extending to adjacent cortical regions. The right SLF is absent and could not be reconstructed (symbolized by the $X$ ), and the left SLF is irregular in appearance (smaller compared with the left SLF from a control).

suggest that the amount of WMT abnormalities is related to the PMG and LIS subgroups and may be interpreted according to the timing of cortical developmental defects, the location and extent of the malformation, and the presence of prenatal CMV infection.

The difference in the extent of WMT abnormalities identified in our LIS and PMG cohorts may reflect the different timing (or targeting) of cortical developmental defects occurring in these 2 MCD classes. Cortical development in humans occurs through a series of orderly, predictable, and overlapping processes. ${ }^{29,30}$ Neuronal proliferation and migration commence early during the late embryonic period and continue throughout the fetal period. Cortical organization through synaptogenesis and dendritic differentiation commences later during the fetal period and continues after birth. ${ }^{31,32}$ The development and maturation of WMTs occur through a similar orderly process. ${ }^{33,34}$ Many association WMTs, including the CG, IFOF, SFOF, and geniculocortical projections (early OR), can be identified as early as at the midfetal period. The ILF and SLF are visible later during the early preterm period and continue to mature during the late preterm period.

According to the classification of Barkovich et al, ${ }^{1}$ cortical developmental defects occur much earlier for LIS (during neuronal migration stage) than PMG cases (during postmigration stage). This may explain the greater extent and severity of WMT abnormalities observed in our LIS sample and the severe intellectual 

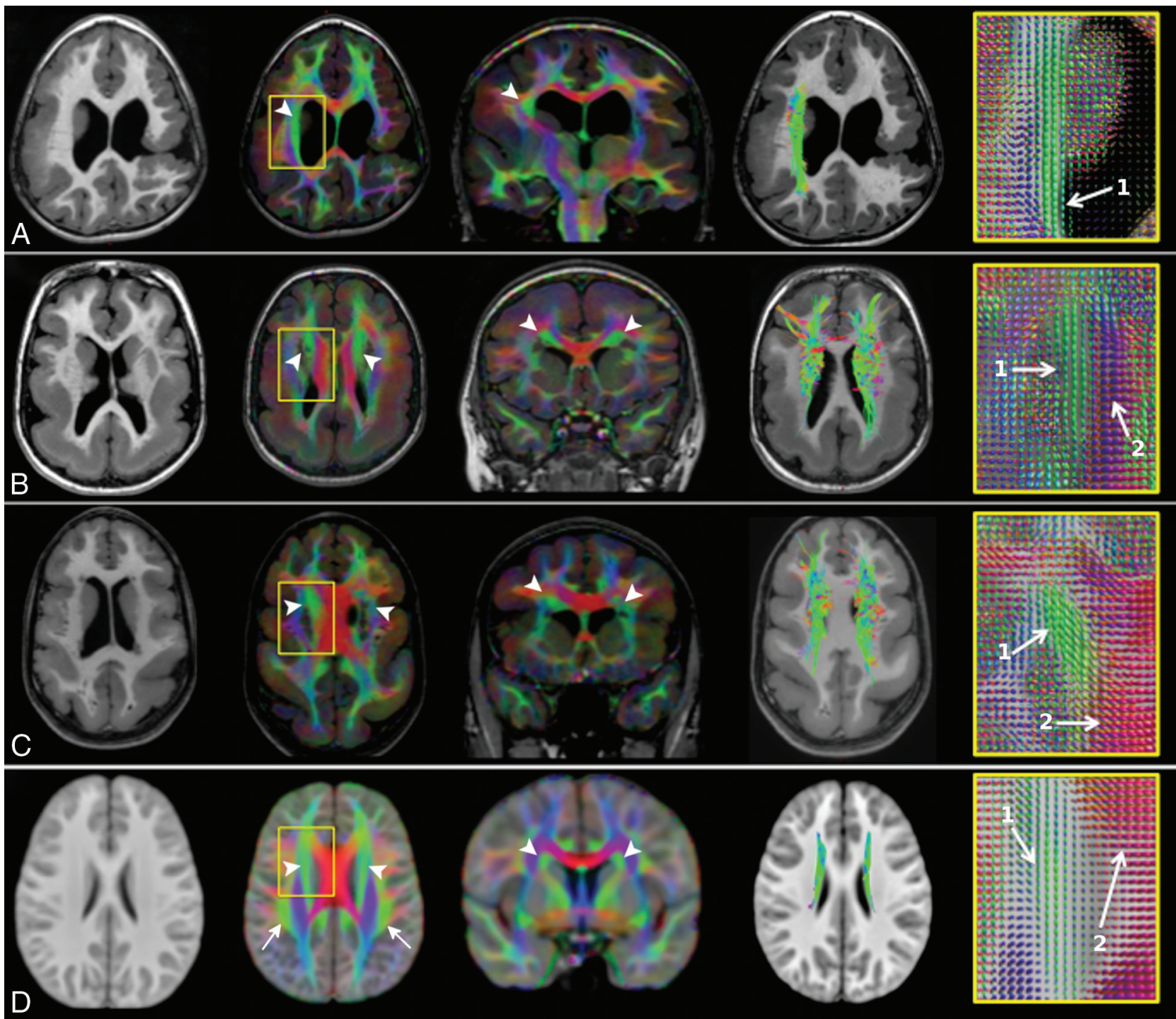

FIG 3. Thickened superior fronto-occipital fasciculus. TIWI, direction-encoded color maps with associated fiber orientation distribution glyph profiles, and tractography reconstructions in 3 patients with thickened SFOFs $(A-C)$ and in the age-matched healthy control template for comparison (D). Patient A has bilateral peri-Sylvian PMG plus a left schizencephaly and a thickened right SFOF (arrowheads). The left SFOF cannot be recognized. Patients $B$ and C demonstrate pachygyria and subcortical band heterotopia and bilateral thickened SFOF (arrowheads). On FOD glyphs maps, 1 indicates the SFOF (green, predominately anterior-posterior oriented fibers), and 2, the corpus callosum (red, predominately leftright oriented fibers).

disability, epilepsy, and cognitive deficits frequently observed in the children with LIS. ${ }^{35}$

The exception to this observation is the widespread WMT abnormalities identified in generalized PMG. A greater proportion of the early-developing WMTs (eg, absent SFOF; irregularappearing CG and IFOF) demonstrated structural abnormalities in these patients compared with other PMG classes. This finding suggests that differential timing (or targeting) of cortical developmental defects may also exist for different PMG classes.

Our findings also suggest that the MCD severity and location can affect the extent of WMT abnormalities. For example, we identified more extensive WMT abnormalities in the generalized PMG than in focal PMG. In our frontal PMG cohort, we observed a frequent association with structurally abnormal CG and SLF, both of which have frontal fiber projections. A recent structural connectivity study using gyral topology-based analysis revealed that altered connectivity in patients with PMG may affect brain regions remote from the primary malformation site. ${ }^{16}$ The presence of altered WMT connections within and between normal-appearing cortices could be secondary to the WMT abnormalities present within the malformed regions. The presence of PCR-OR abnormalities supports visual impairment being observed in up to $25 \%$ of patients with PMG, irrespective of the MCD location. ${ }^{36,37}$

Abnormal SLF morphology was frequently identified in our PMG cohort, consistent with findings from other studies. ${ }^{13,15,17}$ In our peri-Sylvian PMG cohort, the severity of SLF abnormalities (ie, from normal to irregular to absent) was found to be associated with the severity and extent of MCD. Related volumetric analysis (On-line Fig 4) highlighted that the volume of 
malformed cortices in the patients with peri-Sylvian PMG with absent or irregular SLF was significantly higher than that in patients with normal SLFs. This relationship was not investigated for the other WMTs. This finding may have prognostic implications, predicting a higher likelihood of language deficits in the more diffuse form of peri-Sylvian PMG. ${ }^{15}$

In the 5 patients with PMG with diffuse malformations relating to congenital CMV infection, we observed less severe patterns of SLF abnormalities, compared with the remaining PMG cases without CMV infection. While this finding is contrary to our previously stated observation, this preliminary finding implies that the CMV infection affects cortical development in MCD via a distinct mechanism. Previous neuropathologic studies of CMVassociated brain lesions demonstrated preferential tissue destruction within the cerebral cortex and peculiar vulnerability of the radial glia and stem cells. ${ }^{38}$ Accordingly, this finding would explain less severe patterns of WMT abnormalities observed in our CMV-infected patients with PMG, though such speculation warrants further investigation using a larger study cohort.

We observed thickened WMTs (ie, SFOF) in 2 patients with LIS and 1 with PMG. This rare form of WMT structural abnormality has previously been described in a dysmorphic corpus callosum and in aberrant WMTs, such as the supracallosal longitudinal bundle, pontine tegmental cap dysplasia, and anterior mesencephalic cap dysplasia. ${ }^{3,5,7}$ The underlying pathologic mechanism is unclear, though thought to be due to either the transient fetal WMTs failing to regress ${ }^{39}$ or miswiring of WMTs caused by impaired axonal guidance. We additionally observed complete agenesis of the ipsilateral SLF in all 3 cases. Due to the proximity of anatomic courses between the 2 WMTs, we speculate that the thickened SFOF observed in these cases may be due to misguidance of the SLF axonal fibers.

Our study findings are limited by the retrospective study design, multisite MR imaging data, and the limited sample sizes for each PMG and LIS class. Due to the rarity of these disorders, combining multisite cases and MR imaging data is unavoidable. An issue common to all multisite MR imaging studies is a degree of heterogeneity introduced by variations in the MR imaging hardware between sites. Nonetheless, the preliminary analysis we conducted suggested that the interscanner (and intersite) variance had minimal impact on the appearance of the tractography output, which acts to further strengthen the reliability of our study findings. Differences in the DWI acquisition schemes also limit our ability to compare tract-wise diffusion metrics that may be useful to infer changes in the WMT microstructural properties.

The CSD modeling technique used in this study has been shown to reliably estimate local multiple fiber orientations using HARDI data and also the $\mathrm{b} \sim 1100 \mathrm{~s} / \mathrm{mm}^{2}$ and $\sim 30$ diffusionencoded direction data. ${ }^{40}$ Tractography reconstruction may be confounded by operator-dependent bias in the manual ROI placement. We additionally examined the DEC maps and associated FOD glyph profiles to improve the confidence in our tractography findings.

The diagnostic yield of the genetic testing was low in our series $(22 \%)$. The small number of confirmed genetic diagnoses and heterogeneity of the causative genes involved prevented us from characterizing the association between different WMT abnormalities and specific genetic mutations.

\section{CONCLUSIONS}

This is the first dMRI tractography study using an advanced multifiber DWI modeling technique to demonstrate WMT structural abnormalities in PMG and LIS. We demonstrated the following: 1) The brain structural abnormalities in MCD extend beyond the gray matter involvement; 2) patterns of WMT involvement are related to the MCD types, severity, and distribution and possibly to prenatal CMV infection; and 3) there is a spectrum of WMT abnormalities in PMG and LIS, including a rare occurrence of thickened WMTs.

\section{ACKNOWLEDGMENTS}

We would like to thank Dr Andrea Righini and the Radiology Department of V. Buzzi Children's Hospital (Milan, Italy) for the acquisition of DWI data for the traveling healthy control on a $1.5 \mathrm{~T}$ scanner.

Disclosures: Filippo Arrigoni-RELATED: Grant: Australian government, Italian Ministry of Health, Comments: I was funded by the Australian Government through the Endeavour Leadership Program to spend 6 months in Australia (at Murdoch Children's Research Institute, Melbourne) to complete my research. My living costs during my fellowship in Australia were partially covered by an Italian foundation (Fondazione Banco del Monte di Lombardia). My home Institution (E. Medea Scientific Institute, Bosisio Parini, Italy) received money from the Italian Ministry of Health through the "Ricerca Corrente" program for this research; UNRELATED: Employment: E. Medea Scientific Institute, Comments: I work as full-time neuroradiologist at E. Medea Scientific Institute (Bosisio Parini, Italy); Grants/Grants Pending: Ministry of Health, Italian Association for Research on Cancer, and so forth, Comments: I am participating in several research projects at my home Institution (E. Medea Institute, Bosisio Parini, Italy), which are funded by different public and private agencies like the Italian Ministry of Health, or Italian Association for Research on Cancer.* *Money paid to the institution.

\section{REFERENCES}

1. Barkovich AJ, Guerrini R, Kuzniecky RI, et al. A developmental and genetic classification for malformations of cortical development: update 2012. Brain 2012;135:1348-69 CrossRef Medline

2. Basser PJ, Mattiello J, LeBihan D. MR diffusion tensor spectroscopy and imaging. Biophys J 1994;66:247-54 CrossRef Medline

3. Arrigoni XF, Romaniello XR, Peruzzo XD, et al. Anterior mesencephalic cap dysplasia: novel brain stem malformative features associated with Joubert syndrome. AJNR Am J Neuroradiol 2017;38: 2385-90 CrossRef Medline

4. Arrigoni F, Romaniello R, Peruzzo D, et al. The spectrum of brainstem malformations associated to mutations of the tubulin genes family: MRI and DTI analysis. Eur Radiol 2019;29:770-82 CrossRef Medline

5. Caan MWA, Barth PG, Niermeijer J-M, et al. Ectopic peripontine arcuate fibres, a novel finding in pontine tegmental cap dysplasia. Eur J Paediatr Neurol 2014;18:434-38 CrossRef Medline

6. Rollins NK, Booth TN, Chahrour MH. Variability of ponto-cerebellar fibers by diffusion tensor imaging in diverse brain malformations. J Child Neurol 2017;32:271-85 CrossRef Medline

7. Arrigoni F, Romaniello R, Peruzzo D, et al. Aberrant supracallosal longitudinal bundle: MR features, pathogenesis and associated clinical phenotype. Eur Radiol 2016;26:2587-96 CrossRef Medline

8. Poretti A, Meoded A, Rossi A, et al. Diffusion tensor imaging and fiber tractography in brain malformations. Pediatr Radiol 2013; 43:28-54 CrossRef Medline 
9. Huisman T, Bosemani T, Poretti A. Diffusion tensor imaging for brain malformations: does it help? Neuroimaging Clin N Am 2014; 24:619-37 CrossRef Medline

10. Kao YC, Peng SS, Weng WC, et al. Evaluation of white matter changes in agyria-pachygyria complex using diffusion tensor imaging. J Child Neurol 2011;26:433-39 CrossRef Medline

11. Rollins N, Reyes T, Chia J. Diffusion tensor imaging in lissencephaly. AJNR Am J Neuroradiol 2005;26:1583-86 Medline

12. Andrade CS, Leite CC, Otaduy MCG, et al. Diffusion abnormalities of the corpus callosum in patients with malformations of cortical development and epilepsy. Epilepsy Res 2014;108:1533-42 CrossRef Medline

13. Andrade CS, Figueiredo KG, Valeriano C, et al. DTI-based tractography of the arcuate fasciculus in patients with polymicrogyria and language disorders. Eur J Radiol 2015;84:2280-86 CrossRef Medline

14. Lim CCT, Yin H, Loh NK, et al. Malformations of cortical development: high-resolution MR and diffusion tensor imaging of fiber tracts at 3T. AJNR Am J Neuroradiol 2005;26:61-64 Medline

15. Paldino MJ, Hedges K, Gaab N, et al. Failure to identify the left arcuate fasciculus at diffusion tractography is a specific marker of language dysfunction in pediatric patients with polymicrogyria. Behav Neurol 2015;2015:1-9 CrossRef Medline

16. Im K, Paldino MJ, Poduri A, et al. Altered white matter connectivity and network organization in polymicrogyria revealed by individual gyral topology-based analysis. Neuroimage 2014;86:182-93 CrossRef Medline

17. Paldino MJ, Hedges K, Golriz F. The arcuate fasciculus and language development in a cohort of pediatric patients with malformations of cortical development. AJNR Am J Neuroradiol 2016;37: 169-75 CrossRef Medline

18. Dick AS, Tremblay P. Beyond the arcuate fasciculus: consensus and controversy in the connectional anatomy of language. Brain 2012; 135:3529-50 CrossRef Medline

19. Tuch DS, Reese TG, Wiegell MR, et al. High angular resolution diffusion imaging reveals intravoxel white matter fiber heterogeneity. Magn Reson Med 2002;48:577-82 CrossRef Medline

20. Tournier JD, Calamante F, Connelly A. Robust determination of the fibre orientation distribution in diffusion MRI: non-negativity constrained super-resolved spherical deconvolution. Neuroimage 2007;35:1459-72 CrossRef Medline

21. Farquharson S, Tournier JD, Calamante F, et al. White matter fiber tractography: why we need to move beyond DTI. J Neurosurg 2013; 118:1367-77 CrossRef Medline

22. Walker L, Chang L, Nayak A, et al; Brain Development Cooperative Group. The diffusion tensor imaging (DTI) component of the NIH MRI study of normal brain development (PedsDTI). Neuroimage 2016;124:1125-30 CrossRef Medline

23. Pierpaoli C, Walker L, Irfanoglu MO, et al. An integrated software package for processing of diffusion MRI data. In: Proceedings of the Annual Meeting of the International Society of Magnetic Resonance in Medicine, Stockholm, Sweden. May 1-7, 2010:1597

24. Dhollander T, Raffelt D, Connelly A. Unsupervised 3-tissue response function estimation from single-shell or multi-shell diffusion MR data without a co-registered T1 image. In: Proceedings of the Annual Meeting of the International Society of Magnetic Resonance in Medicine Workshop on Breaking the Barriers of Diffusion MRI, Lisbon, Portugal. September 11-16, 2016:5

25. Catani M, Thiebaut de Schotten M. A diffusion tensor imaging tractography atlas for virtual in vivo dissections. Cortex 2008;44:110532 CrossRef Medline

26. Jeurissen B, Tournier JD, Dhollander T, et al. Multi-tissue constrained spherical deconvolution for improved analysis of multishell diffusion MRI data. Neuroimage 2014;103:411-26 CrossRef Medline

27. Jenkinson M, Bannister P, Brady $\mathrm{M}$, et al. Improved optimization for the robust and accurate linear registration and motion correction of brain images. Neuroimage 2002;17:825-41 CrossRef Medline

28. Widjaja E, Blaser S, Miller E, et al. Evaluation of subcortical white matter and deep white matter tracts in malformations of cortical development. Epilepsia 2007;48:1460-69 CrossRef Medline

29. Lui JH, Hansen DV, Kriegstein AR. Development and evolution of the human neocortex. Cell 2011;146:18-36 CrossRef Medline

30. Dubois J, Dehaene-Lambertz G, Kulikova S, et al. The early develop ment of brain white matter: a review of imaging studies in fetuses, newborns and infants. Neuroscience 2014;276:48-71 CrossRef Medline

31. Kostović I, Sedmak G, Judaš M. Neural histology and neurogenesis of the human fetal and infant brain. Neuroimage 2019;188:743-73 CrossRef Medline

32. Bystron I, Blakemore C, Rakic P. Development of the human cerebral cortex: Boulder Committee revisited. Nat Rev Neurosci 2008 9:110-22 CrossRef Medline

33. Vasung L, Raguz M, Kostovic I, et al. Spatiotemporal relationship of brain pathways during human fetal development using high-angular resolution diffusion MR imaging and histology. Front Neurosci 2017;11:1-16 CrossRef Medline

34. Huang H, Xue R, Zhang J, et al. Anatomical characterization of human fetal brain development with diffusion tensor magnetic resonance imaging. J Neurosci 2009;29:4263-73 CrossRef Medline

35. Donato N, Di Chiari S, Mirzaa GM, et al. Lissencephaly: expanded imaging and clinical classification HHS Public Access. Am J Med Genet A 2017;173:1473-88 CrossRef Medline

36. Leventer RJ, Jansen A, Pilz DT, et al. Clinical and imaging heterogeneity of polymicrogyria: a study of $\mathbf{3 2 8}$ patients. Brain 2010;133. 1415-27 CrossRef Medline

37. Kelly JP, Ishak GE, Phillips JO, et al. Visual sensory and ocular motor function in children with polymicrogyria: relationship to magnetic resonance imaging. J AAPOS 2016;20:37-43 CrossRef Medline

38. Teissier N, Teissier N, Fallet-Bianco C, et al. Cytomegalovirusinduced brain malformations in fetuses. J Neuropathol Exp Neurol 2014;73:143-58 CrossRef Medline

39. Kier EL, Fulbright RK, Bronen RA. Limbic lobe embryology and anatomy: dissection and MR of the medial surface of the fetal cerebral hemisphere. AJNR Am J Neuroradiol 1995;16:1847-53 Medline

40. Calamuneri A, Arrigo A, Mormina E, et al. White matter tissue quantification at low b-values within constrained spherical deconvolution framework. Front Neurol 2018;9:1-14 CrossRef Medline 